\title{
Duración de la estancia hospitalaria. Metodologías para su intervención*
}

\section{Length of the Hospitalization. Methodologies for Intervention}

\section{Duração da estancia hospitalar. Metodologias para sua intervenção}

Fecha de recepción: 29-04-14 Fecha de aceptación: 09-05-14 Disponible en línea: 01-07-14 doi: 10.11144/Javeriana.rgyps13-27.dehm

Cómo citar este artículo:

Ceballos-Acevedo T, Velásquez-Restrepo PA, Jaén-Posada JS. Duración de la estancia hospitalaria. Metodologías para su intervención. Rev. Gerenc. Polít. Salud. 2014; 13(27): 274-295. http://dx.doi. org/10.11144/Javeriana.rgyps13-27.dehm

Artículo de revisión, se deriva del proyecto Diseño de políticas para la reducción de la estancia hospitalaria de la IPS universitaria, Sede Clínica León XIII (investigación en curso, fecha de inicio: agosto del 2012, entidad financiadora: IPS Universitaria).

** Investigadora principal, magíster en Logística Integral - Logística Hospitalaria, analista de gestión organizacional, IPS Universitaria, sede Clínica León XIII. Correo electrónico: taticeba@gmail.com. Correspondencia: Calle 30 \# 85-08, barrio Belén Los Alpes, Medellín, Colombia.

*** Coordinadora de Gestión Organizacional de la IPS Universitaria, sede Clínica León XIII, magíster en Ingeniería -

$274^{* * * *} \quad \begin{aligned} & \text { Logística Hospitalaria. Correo electrónico: paulavelasquezr@gmail.com } \\ & \text { Docente del Departamento de Ingeniería Industrial de la Universidad de Antioquia, doctor en Ingeniería de Sistemas. }\end{aligned}$ Correo electrónico: juan.jaen@udea.edu.co 


\section{Resumen}

La estancia hospitalaria prolongada constituye una preocupación mundial, ya que genera efectos negativos en el sistema de salud como, por ejemplo: aumento en los costos, deficiente accesibilidad a los servicios de hospitalización, saturación de las urgencias y riesgos de eventos adversos. El presente trabajo presenta una investigación que enumera las causas más comunes encontradas en la prolongación de la estancia y las metodologías de logística hospitalaria más aplicadas para su estudio y mejoramiento. Resultados: los factores causales de estancias prolongadas más encontrados en la literatura son: demora en la realización de procedimientos quirúrgicos y diagnósticos, necesidad de atención en otro nivel de complejidad, situación sociofamiliar y edad del paciente. Se concluye que para analizar el problema de la estancia hospitalaria es conveniente realizar un "ajuste por riesgo", utilizando el método de grupo relacionado de diagnóstico (GRD) y que una metodología adecuada es la simulación, para la identificación de cuellos de botella.

Palabras clave: estancia hospitalaria; grupo relacionado de diagnóstico; flujo del paciente; logística hospitalaria; gestión de camas; procesos asistenciales

\section{Abstract}

Prolonged hospitalization constitutes a grave concern worldwide because it generates negative effects on the health system such as, for example: increase in costs, deficient accessibility to hospitalization services, overload of emergency services, and the risk of adverse effects. This work presents a research that lists the most common causes found in the extension of the hospitalizations and the most applied hospital logistic methodologies for its study and improvement. Results: The causal agents of extended hospitalizations found with higher frequency in literature are: delay in the performance of surgical and diagnostic procedures; need for attention in another complexity level; social-family situation, and the age of the patient. We conclude that to analyze the problem of hospitalizations it is convenient to perform an "adjustment for risk", using the method of the related diagnosis group (GRD), and simulations are an appropriate methodology to identify bottlenecks.

Keywords: hospitalization; related diagnosis group; patient flow; hospital logic; bed management; medical care processes

\section{Resumo}

A estancia hospitalar prolongada constitui uma preocupação mundial pois gera efeitos negativos no sistema de saúde como, por exemplo: aumento nos custos, deficiente acessibilidade aos serviços de hospitalização, saturação das urgências e risco de eventos adversos. O presente trabalho apresenta pesquisa que enumera as causas mais comuns encontradas na prolongação da estância e as metodologias de logística hospitalar mais aplicadas para o seu estudo e melhoramento. Resultados: os fatores causais de estâncias prolongadas mais encontrados na literatura são: demora na realização de procedimentos cirúrgicos e diagnósticos, necessidade de atendimento em outros níveis de complexidade, situação sociofamiliar e idade do paciente. Conclui-se que para analisar o problema da estância hospitalar é conveniente realizar um "ajuste por risco", utilizando o método de grupos de diagnósticos relacionados (DRG) e que uma metodologia adequada é a simulação, para a identificação de engarrafamentos.

Palavras-chave: estância hospitalar; grupos de diagnósticos relacionados; fluxo do paciente; logística hospitalar; gestão de leitos; processos assistenciais 


\section{Introducción}

En los países latinoamericanos, la desproporción entre oferta y demanda de servicios de salud es un importante motivo de preocupación (1). En el caso de Colombia, el sistema de salud atraviesa una crisis por la incontenible demanda de los servicios de salud, que supera la capacidad de los hospitales para atender pacientes (2). Esta situación se ve también reflejada en la saturación de los servicios de urgencias, al punto de que se presentan casos de cierre temporal de estos (3). Teniendo en cuenta que los servicios de urgencia son la principal puerta de entrada a la hospitalización (entre 70 y $75 \%$ de los ingresos hospitalarios) (4), es común que cuando los pacientes llegan a un hospital no haya disponibilidad de cama para atender su necesidad (3).

Ante la desproporción entre oferta y demanda de servicios de salud, que conlleva la saturación de los servicios, la eficiencia es una condición importante debido a que los recursos son limitados. En los hospitales la cama es uno de los recursos centrales para la prestación de servicios. Por eso, entre las medidas globales de la eficiencia hospitalaria están aquellas que se relacionan con el uso que se le dé a este recurso. El promedio de la estancia hospitalaria de pacientes es utilizado como indicador de eficiencia, porque resume el aprovechamiento de la cama y la agilidad de los servicios prestados en los hospitales (5).

De este modo, un servicio de hospitalización con estancias prolongadas de pacientes pueden considerarse como indicador de falta de eficiencia en el flujo de pacientes. Además, de la relación directa entre la prolongación de la estancia y la disponibilidad de camas, también se aumentan los costos de 276 la atención, el riesgo de eventos adversos y la mortalidad (6).
La estancia prolongada de pacientes en hospitalización es un problema que afecta a instituciones prestadoras de servicios de salud (IPS), a aseguradoras y a pacientes porque:

- Limita la capacidad de los hospitales, dificultando el acceso de pacientes a una cama hospitalaria. Al presentarse mayor estancia, hay menor disponibilidad de cama y mayor saturación en los servicios de urgencias por la espera de pacientes por cama (7). Se suma a esta situación el déficit de camas, un problema común en la capacidad de atención de los hospitales en países como Colombia, Argentina, Brasil y México (8), según la encuesta del Departamento de Desarrollo Humano del Banco Mundial del 2007.

- Incrementa los costos de operación por el uso no apropiado de los recursos. La estancia prolongada implica un desperdicio o mala utilización de los recursos económicos y humanos, ya que lleva a costos adicionales en términos de personal, alimentación, insumos, uso de equipos y utilización de recursos extras del hospital $(7,9,10)$.

- Afecta la calidad de la atención, en términos de seguridad del paciente. El hecho de que se incremente la duración de la estancia, hace que se suba la probabilidad de que un paciente sufra un evento adverso, y especialmente de contraer infecciones nosocomiales, que pueden llevar a la muerte (7, 9-13). Asimismo, el hecho de que un paciente sufra un evento adverso, puede llevar a un aumento de la estancia hospitalaria.

Ante esto, las instituciones de salud tienen la necesidad de reducir la estancia hospitalaria para aumentar el flujo de pacientes a través del logro de mayores niveles de eficiencia 
en la operación, sin afectar la calidad de la atención. Según el grupo de investigación de Advisory Board Company, los hospitales que logran reducir las estancias prolongadas de pacientes pueden agregar camas, aumentando así la capacidad efectiva (14), como se muestra en la tabla 1.

Entonces, para un hospital de trescientas camas (en promedio), reducir la duración de la estancia en un día puede ser el equivalente a contar con 49 nuevas camas en la institución, lo cual es un impacto significativo para la accesibilidad de pacientes a los servicios de hospitalización, reducción de la saturación de los servicios de urgencias y generación de ganancias para los hospitales.

Dado este contexto, se hace pertinente preguntar: ¿cuáles son los factores más frecuentemente identificados en la literatura que inciden en la prolongación de la estancia hospitalaria de los pacientes?, ¿qué metodologías se han implementado para intervenirla? Y iqué resultados se han logrado con la aplicación de estas metodologías en los hospitales?

El propósito del presente trabajo consiste en responder a estas preguntas, a partir de una revisión de la literatura. En la siguiente sección se presentará la metodología em- pleada para la búsqueda de los artículos que permitan analizar la estancia hospitalaria. Posteriormente, se presentan los resultados de la investigación donde se identifican factores que influyen en la prolongación de estancia hospitalaria, aspectos que se deben tener en cuenta para analizar la estancia y las metodologías con las que se ha intervenido. Finalmente, se establece la discusión y las conclusiones de la investigación.

\section{Métodos}

Esta revisión se realizó a través de buscadores genéricos de Internet y bases de datos bibliográficas: Dialnet, Science Direct, Lilacs, Scielo, Jstor, Medline y consulta de páginas especializadas.

La metodología de búsqueda de factores de prolongación de la estancia requirió la identificación de trabajos que cumplieran dos condiciones fundamentales: 1) que plantearan problemas de tiempos de estancia o flujo de pacientes en hospitales, 2) que los estudios identificaran y concluyeran las variables y factores relevantes del problema. Se identificó un conjunto de términos de referencia para abarcar los criterios de inclusión: prolongación de la estancia hospitalaria, inadecuación de la estancia hospitalaria, flujo de pacientes

TABLA 1. Ganancia “EFectiva” de Camas a PaRTiR de la Reducción de la duRación de la estancia HOSPITALARIA

\begin{tabular}{|l|c|c|c|c|c|}
\hline & \multicolumn{5}{|c|}{ Tamaño del hospital (camas) } \\
\hline $\begin{array}{l}\text { Duración de la internación } \\
\text { Reducción (días) }\end{array}$ & $\mathbf{2 0 0}$ camas & $\mathbf{3 0 0}$ camas & $\mathbf{4 0 0}$ camas & $\mathbf{5 0 0}$ camas & $\mathbf{6 0 0}$ camas \\
\hline 0,25 días & 8 & 12 & 16 & 20 & 25 \\
\hline 0,50 días & 16 & 25 & 33 & 41 & 49 \\
\hline 0,75 días & 25 & 37 & 49 & 61 & 74 \\
\hline 1,00 día & 33 & 49 & 65 & 82 & 98 \\
\hline 1,25 días & 41 & 61 & 82 & 102 & 123 \\
\hline 1,50 días & 49 & 74 & 98 & 123 & 147 \\
\hline
\end{tabular}

Fuente: (14) 
y duración de la estancia hospitalaria (y su homólogo en inglés, length of stay (LOS)). Se seleccionaron 34 artículos que cumplieron con los criterios de selección, publicados entre los años 1984 y 2013 en diferentes países.

En la revisión de las metodologías de investigación para la solución del problema se incluyeron trabajos que cumplieran dos condiciones fundamentales: 1) que los problemas pudieran ser resueltos a partir de metodologías de logística hospitalaria, y 2) que en su solución, la metodología de logística hospitalaria identificara las variables y factores relevantes del problema para la aplicación de la metodología. Aquí los términos utilizados fueron: logística hospitalaria, metodologías cuantitativas en salud, procesos, dinámica de sistemas, simulación de eventos discretos, teoría de restricciones, análisis envolvente de datos, pensamiento esbelto (lean thinking), procesos de Markov. Con estos criterios se seleccionaron 21 artículos publicados entre los años 1998 y 2012.

La información se clasificó en tablas para agrupar los principales factores que prolongan la estancia de pacientes en los hospitales, las metodologías aplicadas, los logros obtenidos con la aplicación de estas y las referencias que tienen características comunes según los criterios de clasificación.

En las siguientes secciones se presentan los resultados de la revisión.

\section{Resultados}

Factores que influyen en la prolongación de estancias hospitalarias

En la revisión de la literatura se encontró que las estancias prolongadas en los hospitales son a causa de factores derivados de los diferentes actores del sistema: personal de salud, administración hospitalaria, pacientes y relación entre entidades de la red de atención. En este trabajo se categorizaron los factores distribuyéndolos según el actor en el sistema, como se muestra en la tabla 2, además de mostrar la bibliografía de acuerdo con el porcentaje de literatura encontrada por tipo de factor identificado.

En los factores asociados al personal de salud, se destacan factores propios relacionados con el criterio y competencia médica y la conciencia con respecto a la estancia del paciente en el hospital (18). Esto se traduce en no prestar atención a la necesidad de generar el alta una vez conseguido el propósito de la hospitalización (15-23). Además, fallas al ingreso del paciente por falta de un diagnóstico claro al ingreso $(18,19,24-27)$ y una admisión prematura $(16,18,19,28-30)$.

Las principales fallas del sistema de salud que afectan la duración de la estancia son: la dificultad de la remisión de pacientes a otro nivel de complejidad cuando así lo requieren $(7,17$, $18,20-22$ 25, 27-29, 31, 33, 34) y la demora en las autorizaciones $(18,27,36)$ por parte de las entidades aseguradoras de salud. Esto evidencia los factores asociados con la falta de la consolidación de una red de atención.

Con referencia a la administración hospitalaria, se observa que la larga estancia de pacientes está asociada a la eficiencia en la interacción del servicio de hospitalización con otros servicios del hospital como son los tiempos de respuesta de los procedimientos quirúrgicos y diagnósticos $(6,7,15-25,29$, $3-34,36,37,39,40,42)$ y la transferencia del paciente entre servicios (41). Además, se encuentran estudios que muestran que el flujo de atención se reduce en los fines de semana, ya que la disponibilidad de personal para la atención de los servicios quirúrgicos y de procedimientos diagnósticos se reduce $(17,18,20,21,24,31,33,38-40)$. 
TABLA 2. REVISIÓN dE LITERATURA DE LOS FACTORES QUE PROLONGAN LA ESTANCIA HOSPITALARIA

\begin{tabular}{|c|c|c|c|}
\hline Atribución & Factor & $\begin{array}{l}\text { Porcentaje de } \\
\text { las referen- } \\
\text { cias }\end{array}$ & Referencias \\
\hline \multirow{6}{*}{$\begin{array}{l}\text { Personal } \\
\text { de salud }\end{array}$} & No prestar atención a la necesidad de dar el alta & $26,5 \%$ & $15-23$ \\
\hline & Falta de diagnóstico claro al ingreso & $17,6 \%$ & $18,19,24-27$ \\
\hline & Admisión prematura & $17,6 \%$ & $16,18,19,28-30$ \\
\hline & Tratamiento médico conservador & $5,9 \%$ & 31,32 \\
\hline & Técnica quirúrgica que puede mejorar & $2,9 \%$ & 30 \\
\hline & $\begin{array}{l}\text { Médicos mantienen pacientes en el hospital } \\
\text { por ganancias monetarias }\end{array}$ & $2,9 \%$ & 21 \\
\hline \multirow{3}{*}{$\begin{array}{l}\text { Relación } \\
\text { entre en- } \\
\text { tidades de } \\
\text { la red de } \\
\text { atención }\end{array}$} & $\begin{array}{l}\text { Necesidad de atención en otro nivel de com- } \\
\text { plejidad }\end{array}$ & $38,2 \%$ & $\begin{array}{c}7,17,18,20,21,22,25 \\
27-29,31,33,34\end{array}$ \\
\hline & $\begin{array}{l}\text { Procedimientos diagnósticos o terapéuticos } \\
\text { que pueden realizarse ambulatoriamente }\end{array}$ & $32,4 \%$ & $10,15-20,26,32,34,35$ \\
\hline & $\begin{array}{l}\text { Demora en las autorizaciones por parte del } \\
\text { asegurador }\end{array}$ & $8,8 \%$ & $18,27,36$ \\
\hline \multirow{3}{*}{$\begin{array}{l}\text { Adminis- } \\
\text { tración } \\
\text { hospita- } \\
\text { laria }\end{array}$} & $\begin{array}{l}\text { Demora en la realización de procedimientos } \\
\text { quirúrgicos y diagnósticos }\end{array}$ & $67,6 \%$ & $\begin{array}{l}6,7,15-25,29,31-34,36 \\
37-40,42\end{array}$ \\
\hline & Día de la semana & $29,4 \%$ & $\begin{array}{c}17,18,20,21,24,31,33 \\
38-40\end{array}$ \\
\hline & Transferencia de pacientes entre unidades & $2,9 \%$ & 41 \\
\hline \multirow{4}{*}{ Paciente } & Edad & $38,2 \%$ & $11,17-20,26,27,30,39-43$ \\
\hline & Situación socio-familiar & $38,2 \%$ & $\begin{array}{c}7,11,17,18,22,23,25,27 \\
29-31,39,40\end{array}$ \\
\hline & Condición clínica del paciente & $32,4 \%$ & $17,18,25,26,27,40-45$ \\
\hline & Sexo & $11,8 \%$ & $19,27,40,43$ \\
\hline Otro & Ocurrencia de evento adverso & $20,6 \%$ & $6,11,18,27,38,40,44$ \\
\hline
\end{tabular}

Fuente: elaboración propia

La duración de la estancia hospitalaria, también se ve afectada por características del paciente. Su situación socio-familiar condiciona el apoyo a los cuidados en su domicilio, el rechazo de la familia a aceptar el traslado a un hogar de ancianos, o las condiciones inadecuadas de las viviendas $(7,11,17,18$, $22,23,25,27,29-31,39,40)$.

Otro factor que lleva a un aumento de la estancia hospitalaria es la ocurrencia de eventos adversos, los cuales pueden ser atribuibles a la atención de salud o a las características de los pacientes $(6,11,18,27,38$, 40, 44). Por ejemplo, Palacios y Bareño (11), encontraron que en instituciones de salud adscritas a una aseguradora en Antioquia y el Eje Cafetero (Manizales, Risaralda y Quindío), al 60,5\% de los pacientes con evento adverso (EA) se les incrementó la estancia. Además, un estudio realizado en España (11) muestra que $31,4 \%$ de los pacientes con EA presentaron incremento de la estancia de once días, frente a los que no tuvieron, cuya mediana fue de cuatro días. También se evidencia que un paciente con estancia prolongada tiene mayor probabilidad de 279 sufrir un evento adverso. 
Según esta revisión, se concluye que el factor más frecuentemente encontrado en la literatura, que prolonga la estancia en los hospitales es la demora en la realización de procedimientos quirúrgicos y diagnósticos, seguido de la necesidad de atención en otro nivel de complejidad, de la situación sociofamiliar y la edad del paciente.

La identificación de estos factores muestra que la estancia hospitalaria prolongada es un problema complejo, de ahí que los hospitales y la Academia lo consideren un problema de estudio de amplio interés. Como la estancia hospitalaria no solo está relacionado con problemas en la eficiencia de la administración hospitalaria, sino también con las características de los pacientes, se hace necesario recurrir a un método de "ajuste por riesgo" de la estancia, para determinar cuándo se incurre en estancia prolongada por problemas de la atención.

\section{Ajuste por riesgo de la estancia hospitalaria}

El ajuste por riesgo consiste en el control de las variables que reflejan las características de los pacientes, lo cual es necesario para atribuir diferencias a problemas de atención. Realizar ajuste por riesgo significa contemplar en las comparaciones, las diferencias entre pacientes en cuanto al riesgo de alcanzar determinado resultado que no dependen de la calidad o eficiencia de los servicios prestados (5).

Existen distintos métodos que han sido utilizados para el ajuste de riesgos, como son los grupos relacionados de diagnóstico (GRD) (el más utilizado mundialmente) $(43,46)$, el sistema de clasificación por estadío (conocido como Staging), el índice de Horn, el índice de Salemi y el Apache, específico para unidades de cuidados intensivos, entre otros (5).
Los GRD surgen en Estados Unidos durante la década de los sesenta en la Universidad de Yale, propuestos por Fetter y Thompson (46) como uno de los primeros intentos por clasificar a los pacientes de modo que se obtuvieran grupos homogéneos en cuanto a costos hospitalarios. Estos grupos fueron conformados por un panel de expertos basado en cinco aspectos del paciente: el diagnóstico principal, la intervención quirúrgica, la edad, las complicaciones y el motivo del alta. Como se necesitó paneles de expertos para construir las combinaciones de los diagnósticos similares, tanto en términos clínicos como de consumo, el método ha sido implementado en software comerciales (5).

Los GRD han resultado útiles para estimar el costo medio del tratamiento de los pacientes que pertenecen a una misma categoría; el estudio de la casuística o case mix; la comparación entre hospitales; la evaluación de diferencias en las tasas de mortalidad de los pacientes hospitalizados; y la ejecución y el soporte de guías clínicas que disminuyan la variabilidad en la práctica clínica y la estancia media hospitalaria. Por lo tanto, se constituyen en una herramienta que sirve para el control de la calidad y la facturación de la atención, y como insumo para la planificación de la administración hospitalaria (23).

Específicamente en el marco de la logística hospitalaria, se han venido desarrollando estudios cuyos objetivos son la reducción de costos, la eficiencia en el uso y asignación de los recursos, la optimización de los flujos en los sistemas, la mejora en la calidad del servicio y la difusión de sistemas de soporte que integren la información que se genera en el sistema y que es clave en el desarrollo de modelos para la toma de decisiones, ante un panorama de capacidades limitadas y una demanda creciente por los servicios (47). De modo que las metodologías de logística hospitalaria permiten la búsqueda de la efi- 
ciencia en los sistemas de salud, planteando alternativas de solución que sean viables en su implementación.

En la siguiente sección se describen metodologías de la logística hospitalaria utilizadas para orientar la administración hospitalaria en la búsqueda de la eficiencia de las operaciones.

\section{Metodologías para el estudio de alternativas de intervención del proceso de estancia hospitalaria}

La revisión de la literatura permite identificar diferentes metodologías en el marco de la logística hospitalaria para entender y abordar los diferentes factores que prolongan la estancia y los efectos derivados de esta. Estas metodologías se encuentran orientadas a lograr mayores niveles de eficiencia en la administración hospitalaria, como son la simulación de eventos discretos, la dinámica de sistemas, el análisis envolvente de datos, la teoría de restricciones, los modelos de
Markov, el pensamiento esbelto (lean thinking) y la reingeniería.

Velásquez et ál. (48) realizan una extensa revisión de la literatura sobre metodologías cuantitativas para la optimización del servicio de urgencias, de donde se puede concluir que los problemas relacionados con la estancia hospitalaria son el segundo caso de estudio más abordado para explicar la saturación de urgencias (48). De este modo, se incluyen en el estudio de la prolongación de la estancia hospitalaria, las metodologías que abordan directamente la saturación de los servicios de urgencias como consecuencia de largas estancias en hospitalización.

En la tabla 3 se recopilan las metodologías con sus respectivos objetivos de estudio.

A continuación se describen los conceptos que abarcan cada metodología recopilada en la tabla 3, cómo se han aplicado para el

TABLA 3. MÉtodos EMPLEAdOS EN EL ABORDAJE DE PROBLEMAS RELACIONADOS CON LA ESTANCIA PROLONGADA DE PACIENTES

\begin{tabular}{|c|c|c|}
\hline Métodos & Objetivo de estudio & Referencia \\
\hline $\begin{array}{l}\text { Simulación } \\
\text { de eventos } \\
\text { discretos }\end{array}$ & $\begin{array}{l}\text { - } \quad \text { Evaluación de nuevos procesos } \\
\text { Disminuir los tiempos de espera para que los pacientes sean } \\
\text { atendidos } \\
\text { - Mejorar el rendimiento de los procesos asistenciales }\end{array}$ & 49,50 \\
\hline $\begin{array}{l}\text { Dinámica de } \\
\text { sistemas }\end{array}$ & $\begin{array}{l}\text { - } \quad \text { Disminuir los tiempos de espera de los pacientes } \\
\text { - } \quad \text { Realizar mejoras en los flujos de pacientes y la capacidad del sistema } \\
\text { Evaluar los efectos de las propuestas y políticas implementadas } \\
\text { para el mejoramiento de procesos } \\
\text { - Analizar la utilización de camas }\end{array}$ & $51-54$ \\
\hline $\begin{array}{l}\text { Teoría de } \\
\text { restricciones } \\
\text { (TOC) }\end{array}$ & $\begin{array}{l}\text { - Incrementar el número de pacientes tratados en la misma unidad } \\
\text { de tiempo (throughput), sin necesidad de recursos adicionales y sin } \\
\text { aumentar la exigencia o sobrecargar al personal para trabajar más } \\
\text { intensamente. } \\
\text { - Identificar el punto de apalancamiento que explique la razón del } \\
\text { promedio del número de días de hospitalización. } \\
\text { - Reducir el promedio de días de estancia con los mismos recursos. }\end{array}$ & $54-58$ \\
\hline $\begin{array}{l}\text { Análisis en- } \\
\text { volvente de } \\
\text { datos (AED) }\end{array}$ & $\begin{array}{l}\text { - } \quad \text { Evaluar el aumento de la eficiencia técnica } \\
\text { - } \quad \text { Analizar el incremento en el uso de la capacidad instalada. } \\
\text { - } \quad \text { Analizar el incremento en la capacidad de los hospitales. } \\
\text { - Evaluar el cambio en la estancia media por paciente. }\end{array}$ & 59 \\
\hline
\end{tabular}




\begin{tabular}{|l|l|c|}
\hline Métodos & Objetivo de estudio & Referencia \\
\hline $\begin{array}{l}\text { Reingeniería } \\
\text { de procesos }\end{array}$ & $\begin{array}{l}\text { Definición de procesos para asignación de camas } \\
\text { Rediseño de procesos para reducir la variabilidad en el ingreso y } \\
\text { al alta de paciente }\end{array}$ & $60-62$ \\
\hline $\begin{array}{l}\text { Rediseño del proceso de hospitalización } \\
\text { Modelos de }\end{array}$ & $-\quad \begin{array}{l}\text { Predecir la estancia promedio de pacientes en el hospital } \\
\text { Markov }\end{array}$ & $\begin{array}{l}\text { la atención de pacienten } \\
\text { Describir la dinámica estocástica de pacientes }\end{array}$ \\
\hline $\begin{array}{l}\text { Pensamiento } \\
\text { esbelto }\end{array}$ & $-\quad \begin{array}{l}\text { Realizar mejoras en el proceso de hospitalización } \\
\text { Disminuir la duración de la estancia media }\end{array}$ & $63-66$ \\
\hline
\end{tabular}

Fuente: elaboración propia

estudio de la estancia hospitalaria y los logros obtenidos en su implementación.

Simulación de eventos discretos (SED). Una simulación de eventos discretos es aquella en la que los cambios de estado de las variables se realizan en puntos discretos del tiempo accionados por eventos, o dicho de otra manera, sistemas que evolucionan en el tiempo mediante cambios instantáneos en las variables de estado (70). Algunos ejemplos de eventos de simulación son la llegada de un paciente, la falla de un recurso o equipo, la terminación de una actividad y la finalización de un turno de trabajo.

En el sector de la salud, las aplicaciones más frecuentes de SED tienen que ver con programación de la atención, planificación de capacidad, mejora de la productividad, tiempo de ciclo, reducción de costes, capacidad de rendimiento, aprovechamiento de los recursos y la búsqueda de cuellos de botella (49). El resultado de estas aplicaciones se refleja en los flujos de pacientes en las camas de los hospitales, la ocupación de camas y la duración de la estancia.

Günal y Pidd (71) citan un estudio de una simulación de camas en hospitalización para las admisiones de emergencia, concluyendo que el riesgo de una escasez de camas de hospital es bajo cuando la ocupación de camas promedio permanece por debajo del
$85 \%$. Este modelo muestra que las crisis de cama pueden ser debido a la naturaleza de las llegadas estocásticas de los pacientes.

El-Darzi E. et ál. (50) se apoyan en este método para estudiar la ocupación de camas. Su metodología no incluye el uso explícito de SED, sin embargo, en algunos documentos lo utilizan para demostrar cómo se podrían utilizar los modelos matemáticos en la vida real (50). Asimismo (50), estudian el flujo de pacientes en un hospital geriátrico utilizando esta metodología, consideran el problema como un sistema de colas para evaluar el efecto de la obstrucción en el flujo de los pacientes en los departamentos geriátricos. El modelo prevé la duración media de la estancia, así como el número promedio de pacientes en cada estado (50).

Van Berkel y Blake (72) analizan la lista de espera para el servicio de cirugía de un hospital de Canadá, para lo cual desarrollan un modelo de simulación de eventos discretos. El aporte de este modelo es orientar las decisiones para la planificación de la capacidad y analizar el comportamiento de este servicio. $\mathrm{El}$ análisis examinó las consecuencias de la redistribución de camas entre los sitios y el estándar de la estancia hospitalaria. A partir de los resultados, se disminuyó la espera de pacientes para la realización de los procedimientos quirúrgicos. 
Dinámica de sistemas. La dinámica de sistemas es un método de construcción de modelos de sistemas susceptibles de ser simulados por un software especializado para esto. La dinámica de sistemas se remonta a finales de los años cincuenta, gracias al trabajo de Forrester, del Instituto Tecnológico de Massachussets, quien lo utilizó para estudiar los niveles de pedido en la compañía americana donde trabajaba (73). La dinámica de sistemas permite mostrar cómo las políticas, las decisiones, la estructura y los tiempos se interrelacionan para influir en la estabilidad de un sistema (73).

Williams (51) utiliza dinámica de sistemas para estudiar la llegada de pacientes como una manera efectiva de disminuir la espera y el uso promedio de los recursos y hacer que la eficiencia sea menos sensible a las llegadas aleatorias.

Wolstenholme (52) describe la aplicación de la dinámica de sistemas de asistencia sanitaria y social en Europa. Cita un caso de estudio en el Reino Unido, donde se muestra que para reducir la estancia hospitalaria la opción más económica es aumentar la capacidad del hospital, reduciendo con ello el retraso de los egresos y las operaciones electivas mayores, mientras que la opción más costosa es aumentar el número de camas del hospital.

Cooke (53) cita estudios de dinámica de sistemas realizados en el Sistema Nacional de Salud del Reino Unido (NHS), donde el principal beneficio del modelo fue ver el uso de dinámica de sistemas como herramienta de aprendizaje para demostrar que los cambios en los recursos (camas, personal) tuvieron menos impacto que los cambios en los comportamientos que afectan a los patrones de referencia, como la duración de la estancia o los flujos intersectoriales.
Ahmad et ál. (54) proponen un híbrido entre dinámica de sistemas y teoría de restricciones para solucionar la planificación de la capacidad para abordar el tiempo de espera del paciente y los problemas de prestación de servicios en un hospital público de Penang, Malasia.

En conclusión, la dinámica de sistemas permite entender el flujo de pacientes en la estructura de un hospital, entendido este como un sistema. La perspectiva que ofrece la aplicación de esta metodología facilita en gran medida la toma de decisiones en un nivel estratégico y táctico.

La simulación de eventos discretos y la dinámica de sistemas, ambos de la rama de simulación, ofrecen como ventaja que permiten experimentar diferentes decisiones para predecir su impacto. Los resultados indican que un aumento potencial de rendimiento es posible sin recursos adicionales (72).

Teoría de restricciones. La Teoría de Restricciones (TR) es una filosofía de gestión aplicable a cualquier tipo de empresa, que tiene como objetivo mejorar el funcionamiento de los sistemas de gestión de las organizaciones para lograr los objetivos deseados (74-76). Fue creada por el físico israelí Eliyahu Goldratt, quien aplicó la lógica de la física al desarrollo de una propuesta de mejoramiento para empresas productivas y la incluyó en su obra La meta. También ha sido implementada en organizaciones sin ánimo de lucro, como instituciones educativas, hospitales e instituciones militares (75).

La aplicación de TR se realiza de acuerdo con dos principios. El primero de ellos es que la TR está fundamentada en el pensamiento sistémico, que concibe a la organización como un sistema complejo donde todas las partes interactúan entre sí. Y el segundo, es que cada sistema debe tener al menos una restricción 
que limita el logro de mayores y mejores resultados con respecto a la meta (55).

\section{En la obra We All Fall Down: Goldratt's Theory} of Constraints for Healthcare Systems, de Julie Wright y Russ King se ilustra la TR aplicada en salud. La historia está ambientada en un hospital universitario de tamaño medio del Reino Unido y muestra la metodología para liberar más camas para los pacientes, lo cual se logra a través de la TR (56).

En diferentes casos de aplicación de TR se ha logrado incrementar la calidad de la atención de los pacientes, aumentando el número de pacientes atendidos en la misma unidad de tiempo. Esto a su vez ha permitido impactar en la reducción del promedio de días de estancia sin necesidad de recursos adicionales. Algunos ejemplos se presentan en Alcanzando un desempeño superior a través de todo el sistema de salud y seguridad social, obra escrita por A. Knight, de Goldrat Consulting (57):

- El hospital general Milton Keynes incrementó el número de pacientes vistos dentro de las cuatro horas siguientes a la llegada del paciente al servicio de urgencias, donde luego de atenderse a un $70 \%$ de los pacientes, se pudo atender a más del $90 \%$, sin recursos adicionales. Esta mejora, efectuada en el plazo de dieciséis semanas, permitió que la institución se ubicara como uno de los hospitales con mejor desempeño en el servicio de urgencias en los Estados Unidos (57).

- En Oxford, diez hospitales comunitarios lograron reducir su promedio de estancia hospitalaria en un $50 \%$, sin recursos adicionales. Pasaron de un promedio de estancia de 60 días a solo 29 , y casi doblaron el número de pacientes atendidos en un lapso de seis meses (57).
- El hospital John Radcliffe logró reducir el promedio de días de estancia hospitalaria en el área de gerontología entre 50 y $70 \%$ en un periodo de seis meses. Esto le permitió reducir los bloqueos en la admisión de pacientes y liberar capacidad (57).

En Colombia existe un caso documentado de la aplicación de TR con el objetivo de reducir la estancia hospitalaria (58). En el 2007, en el hospital El Tunal de Bogotá hubo un aumento en el throughput, es decir, en el número de pacientes atendidos por unidad de tiempo. Específicamente en el servicio de Ginecología y Obstetricia, se incrementó el $38,6 \%$ con respecto al primer semestre de 2007. En el servicio de pediatría hubo un aumento de 7,5\% en comparación con el 2006, y en el servicio de medicina interna hubo un aumento de 5,2\% en el segundo semestre de 2007. Con la aplicación de TR durante un lapso de cinco meses, se logró incrementar el flujo de pacientes atendidos durante los últimos cuatro meses del año 2007.

En el área de la salud hay muy pocas implementaciones documentadas conocidas (55), pero se ha demostrado que esta es una metodología que rinde resultados en la reducción de la estancia hospitalaria. Breen, Burton-Houle y Aron (55) concluyen que: "La TR ofrece, un riguroso método para analizar y mejorar el funcionamiento de los sistemas de Salud”.

Análisis Envolvente de Datos (AED). Es una herramienta que permite comparar la eficiencia entre diferentes organizaciones que cuenten con características similares. Con esta herramienta se puede determinar las organizaciones que son más eficientes en el desempeño del grupo y calcular los niveles que deben alcanzar las ineficientes para convertirse en eficientes (77). 
TABLA 4. SELECCIÓN DE INPUTS Y OUTPUTS USADOS EN ESTUdIOS AED EN EL SECTOR SALUd

\begin{tabular}{|c|c|c|}
\hline Inputs & Outputs (productos) & Outputs (resultados) \\
\hline $\begin{array}{ll}\text { - } & \text { Número de camas } \\
\text { - } & \text { Número de quirófanos } \\
\text { - } & \text { Número de equipos de RMN } \\
\text { - } & \text { Número de médicos de } \\
\text { - } & \text { plantilla } \\
\text { - } & \text { tastivo del personal facul- } \\
\text { - } & \text { Costos del personal de en- } \\
\text { - } & \text { Costos en medicamentos } \\
\text { - } & \text { Costos en material sanitario } \\
\text { - } & \text { Costos en suministros } \\
\text { - } & \text { Costos en otros bienes y } \\
\text { - } & \text { Corvicios corrientes } \\
\text { inmovilizado del material }\end{array}$ & $\begin{array}{ll}\text { - } & \text { Número de altas } \\
\text { - } & \text { Número de estancias (ajus- } \\
\text { tadas o no) } \\
\text { - Número de UPA (pondera- } \\
\text { das o no) } \\
\text { - Número de UCH (pondera- } \\
\text { das por GRD) } \\
\text { - Número de consultas ex- } \\
\text { ternas (en policlínica del } \\
\text { propio centro) } \\
\text { - Número de urgencias } \\
\text { Número de intervenciones } \\
\text { quirúrgicas convencionales } \\
\text { Número de intervenciones } \\
\text { de cirugía mayor ambu- } \\
\text { latoria } \\
\text { Número de interconsultas } \\
\text { Número de trasplantes }\end{array}$ & $\begin{array}{l}\text { - Tasa de infecciones noso- } \\
\text { comiales } \\
\text { - } \quad \begin{array}{l}\text { Porcentaje de altas por } \\
\text { curación }\end{array} \\
\text { - Tasa de reingresos por ur- } \\
\text { gencias antes de las } 72 \text { horas } \\
\text { - Tasa de infección por herida } \\
\text { quirúrgica } \\
\text { Prevalencia de pacientes } \\
\text { con úlcera por presión } \\
\text { Porcentaje de fallecimien- } \\
\text { tos por todas las causas } \\
\text { Tasa de fallecimientos neo- } \\
\text { natales }\end{array}$ \\
\hline
\end{tabular}

Fuente: (78)

Para el análisis se tiene como medida el índice global de eficiencia de cada unidad productiva analizada, el cual se calcula como la relación por cociente de la suma ponderada de los outputs (resultados obtenidos) y la suma ponderada de los inputs (recursos utilizados para generarlos) (77).

El modelo AED, desarrollado por Charnes, Cooper y Rhodes, es una metodología que se fundamenta en el hecho de que una unidad que emplea menos inputs que otra para producir el mismo volumen de output se puede considerar más eficiente (78). En la tabla 4 se muestran los inputs y outputs más frecuentemente utilizados para el análisis de la eficiencia a través del AED en el sector salud.

En un estudio realizado en España por Prior y Solá en 1996 (59), se utiliza AED para orientar la toma de decisiones estratégicas para la reducción de listas de espera de pacientes.
Esta reducción se encuentra estrechamente relacionada con incrementos en eficiencia y reducciones de la estancia media. Las decisiones estratégicas que se contemplaron fueron: a) aumento de la eficiencia técnica; b) cambio en la estancia media por paciente; c) incremento en el uso de la capacidad instalada; y d) incremento en la capacidad de los hospitales.

En este estudio se tomaron datos de 137 hospitales generales públicos con más de doscientas camas. Se estudiaron los servicios de medicina interna, cirugía, traumatología, obstetricia y ginecología y otras. Los inputs considerados fueron: número de camas instaladas, personal médico, personal de enfermería, personal administrativo y consumos (medicamentos, alimentación, instrumental, ropería, materiales de consumo y reposición y otros). Los outputs considerados fueron: estancias hospitalarias, altas hospitalarias y consultas externas. 
Los resultados indican que para eliminar las listas de espera en medicina interna, los hospitales no tienen que asumir un aumento en sus costos; incrementando su eficiencia podrían reducirlas hasta en un 3,62\%. En el servicio de obstetricia-ginecología, además de incrementarse la eficiencia, se requiere aumentar personal y consumos; si se incrementara la eficiencia, se lograría reducir un $4,09 \%$ las listas, y un 6,11\% si disminuyera la estancia media. En los servicios de cirugía y traumatología, para reducir las listas de espera, se debe asumir un incremento en los costos de los inputs, aumentando la capacidad del hospital, o bien remitir los pacientes a otros hospitales.

En conclusión, los resultados muestran la limitada reducción de listas de espera cuando solo se consigue el aumento en los niveles de eficiencia sin aumentar costos. Asimismo, evidencian el importante efecto de combinar estrategias para mejorar la estancia media y aumentar la utilización de la capacidad instalada, y también indican que la contención de las listas de espera en los servicios de cirugía y traumatología requiere inversión en el incremento de la capacidad de los hospitales.

Para aplicar AED hay limitaciones en cuanto a la información requerida disponible en Colombia con relación a los inputs y los outputs (77). Finalmente, AED es una herramienta de gran utilidad para realizar análisis de eficiencia que orienten la toma de decisiones en el sector de la salud, como reducir la estancia hospitalaria.

Reingeniería de procesos. Según Champy y Hammer (79), se entiende por reingeniería "la revisión fundamental y el rediseño radical de procesos para alcanzar mejoras espectaculares en medidas críticas y contemporáneas 286 de rendimiento, tales como costes, calidad, servicio y rapidez".
La reingeniería consiste en mejorar el desempeño de una organización rediseñando los procesos de forma radical, táctica y operacional. Esta metodología genera beneficios como mejoras en la calidad, en los procesos, en la flexibilidad, reducción de costos y eficiencia y eficacia en las organizaciones (80). Para lograr esto, Champy y Hammer (81) proponen como factor clave de éxito la incorporación de tecnología de la información (ті) a los procesos que generan valor (81).

Osorio y Paredes (80) muestran que en la literatura anglosajona se evidencia el interés por la reingeniería en los hospitales, con afirmaciones como la que indica que más del $60 \%$ del total de hospitales en Estados Unidos han estado implicados en procesos relacionados con la implementación de la reingeniería. Además, entre las reflexiones finales de estos autores se encuentra que el apogeo de la reingeniería se debe a que considera explícitamente a la tecnología de la información como fuente de mejora radical, a diferencia de la teoría de mejoramiento de la calidad total.

La reingeniería en salud busca obtener los mayores beneficios posibles con el menor riesgo para los usuarios y su ambiente, en función de los recursos disponibles (60). A continuación se citan algunos casos de aplicación de la reingeniería en hospitales.

- En España, el Hospital Universitario de Bellvitge (HUB), de alta complejidad, aplicó reingeniería en el proceso del alta hospitalaria durante el año 2008. Entre los beneficios logrados están: incremento del número de pacientes con el alta planificada con 24 horas de antelación. Mientras que en el 2007 esta cifra representaba el $40 \%$, a finales del 2008 se consolidó en más de $80 \%$; incremento en la rotación de pacientes por cama, que pasó de 42 a 44 pacientes 
por cama, debido a la reducción de la estancia de 8,7 a 8,3 días; se redujeron las cancelaciones quirúrgicas por falta de cama; la media de pacientes pendientes de cama pasó de 4,7 a 3,3 pacientes, lo que representa una reducción del $30 \%$. Esto impacta en el tiempo de espera de los pacientes que llegan a urgencias (82).

- En Italia, tres hospitales (Pontedera, Forlí y Forligno) implementaron reingeniería para el rediseño de la logística del flujo de pacientes, con el objetivo de aumentar su productividad, en razón de una utilización adecuada de capital, equipos y recursos humanos. Para este fin realizaron cambios radicales en la disposición física de los hospitales, en la planificación de la capacidad disponible y en los roles organizacionales que apoyan la gestión del flujo de pacientes. Los resultados se observaron en los tres años siguientes al cambio en la logística del flujo de pacientes, lográndose disminuir la media de la estancia hospitalaria, aumentar la tasa de ocupación de camas, aumentar la complejidad (case mix) y reducir el intervalo de sustitución de camas (61) (ver tabla 5).

- En Colombia, durante el año 2008, en un hospital privado de Bogotá se implementó la reingeniería como metodología para abordar la congestión en su departamento de urgencias, debido a la falta de disponibilidad de camas para pacientes que requerían ser hospitalizados (62). Apoyándose en los principios de la reingeniería, este estudio recomendó implementar un software con características diseñadas para permitir el monitoreo de las actividades de todo el hospital y la gestión de los sistemas en cada unidad. De este modo se eliminarían tiempos muertos en la comunicación entre los procesos de asignación

TABLA 5. INDICADORES DE PRODUCTIVIDAD (ANTES Y DESPUÉS DE LA REINGENIERÍA)

\begin{tabular}{|l|c|c|}
\hline & Antes del cambio & Después del cambio \\
\hline Estancia media & & \\
Pontedera & 5,0 & 4,0 \\
Forli & 6,0 & 4,6 \\
Foligno & 6,1 & 6,5 \\
\hline Tasa de ocupación de camas & & \\
Pontedera & $65 \%$ & $82 \%$ \\
Forli & $71 \%$ & $78 \%$ \\
Foligno & $81 \%$ & $84 \%$ \\
\hline Case mix & & 1,61 \\
Pontedera & 1,12 & 1,19 \\
Forli & 0,99 & 1,09 \\
Foligno & 1,07 & 1,7 \\
\hline Intervalo de sustitución de la cama ${ }^{a}$ & \\
Pontedera & 2,8 & 1,5 \\
Forli & 2,5 & 1,2 \\
Foligno & 1,4 & \\
\hline a Número de días que una cama está vacante igual a [(365 x número de camas en el hospital - número \\
total de días de estancia hospitalaria)/ total de egreso de pacientes $)]$
\end{tabular}

Fuente: (61) 
de cama, alistamiento de habitación y salida de pacientes y en disminuir errores en la información. Como beneficios esperados luego de la implementación del nuevo sistema de comunicación a través del software están: en asignación de camas: en lugar de tener diez procesos basados en comunicaciones, tener siete; en alistamiento de habitación: en lugar de tener tres procesos basados en comunicaciones, tener dos. Todo esto debe repercutir en tiempos de proceso más cortos, menor número de subprocesos en los macroprocesos, y reducción de errores humanos en la comunicación.

En conclusión, mediante la implementación de reingeniería de procesos se pueden lograr mejoras significativas en la eficiencia de los hospitales.

Modelos de Markov. Los modelos de Markov permiten estudiar la evolución temporal de cualquier proceso cuyo estado futuro dependa del resultado de la etapa anterior y no de cualquiera de los resultados previos (83). Estos modelos deben su nombre al matemático ruso Andrei Andreevitch Markov (1856-1922) (84). En el campo de investigación de operaciones, estos modelos se usan para describir y predecir el comportamiento de ciertos sistemas bajo condiciones de incertidumbre a través del tiempo (63). Se han utilizado, por ejemplo, para analizar patrones de compra de deudores, para planear necesidades de personal, para modelar dinámica de poblaciones, sistemas de espera, control de inventarios, mantenimiento y reemplazo de equipos, y en apoyo a la toma de decisiones en administración, ingeniería y medicina, entre otros $(63,84)$.

En el sector de la salud, los modelos de Markov se han utilizado desde comienzos del siglo xx para diferentes tipos de investigación, como análisis farmacoeconómicos, estudios predictivos de mortalidad, toma de decisiones en admisión de pacientes y evolución de enfermedades, entre otros (63).

En Brasil, Nadal (64) modeló un proceso de decisión de Markov para las admisiones electivas (no urgentes) de pacientes en un periodo. El propósito de controlar las admisiones de pacientes es promover una utilización más eficiente de los recursos hospitalarios, evitando la ociosidad o el uso excesivo de estos recursos. Como resultado del modelo se generó una política óptima de control de admisión que mantiene el consumo de recursos cerca de los niveles deseados de utilización, mientras se optimiza la desviación de los costos establecidos.

Pegueroy Riquenes (65) aplicaron la cadena de Markov en un centro hospitalario de Santiago de Cuba, con el objeto de determinar con un nivel de probabilidad dado, la estancia promedio de los pacientes intervenidos quirúrgicamente que pasan a la sala de recuperación, lo cual permite conocer los costos hospitalarios del servicio prestado. Como resultado se da a los directivos de la institución una herramienta para la toma de decisiones que contribuye a conocer los costos hospitalarios por estancia y a optimizar los recursos disponibles.

En un hospital de Chile se elaboró un modelo de cadenas de Markov para el estudio de la dinámica de la permanencia de pacientes en una unidad de cuidados intensivos de cardiología, mediante la definición de determinados niveles de gravedad de un paciente (estados) y la obtención de las correspondientes probabilidades de transición entre un nivel de gravedad y otro. La predicción de la duración en la permanencia de los pacientes en una unidad de cuidado intensivo permite planificar adecuadamente la atención de pacientes (63).

En 1998, en un hospital de tercer nivel de complejidad de Medellín, se aplicaron cadenas de Markov para describir la estancia 
hospitalaria estimando el número esperado de pacientes con trauma en los servicios de hospitalización, cirugía y unidad de cuidados intensivos y en la condición de egreso vivo y muerto, luego de ingresar por urgencias. Como resultado del modelo se facilita predecir el número de recursos requeridos (66).

En general, las cadenas de Markov es un método para predecir la estancia hospitalaria de pacientes, lo cual facilita la planeación de recursos requeridos para la atención y la disminución de la estancia.

Pensamiento esbelto. El pensamiento esbelto (PE) es una metodología de gestión que tiene su origen en el lean manufacturing (manufactura esbelta) olean production (producción esbelta). Esta metodología fue desarrollada por Toyota en el año 1950, fundamentada en gran parte en el trabajo de Deming (85). El sistema de producción de Toyota logró aumentar su ventaja competitiva al producir más carros con menos empleados y menos defectos. Para lograrlo se enfocaron en la búsqueda de la eficiencia y en la mejora de la calidad optimizando sus procesos operativos (67).

El PE tiene como filosofía ofrecer bienes y servicios ajustados a los deseos de los clientes con mayor rapidez, con bajos costos y sin desperdicios. De este modo, esta metodología busca la eliminación de mudas o desperdicios de los procesos, con el fin de crear valor.

El PE considera desperdicios por sobreproducción, tiempo de espera, transporte, exceso de procesado, inventario, movimiento y defectos. Estos desperdicios, además de presentarse en fábricas, se encuentran en empresas del sector de servicios (85). En la tabla 6 se pueden observar algunos ejemplos de desperdicios identificados en los hospitales, bajo este enfoque (67).

TABLA 6. EJEMPLOS DE DESPERDICIOS EN EL SECTOR SALUD

\begin{tabular}{|c|c|}
\hline Desperdicios originales & Ejemplos de desperdicios en salud (NHSIII, 2007) \\
\hline Transporte & $\begin{array}{l}\text { Transporte: } \\
\text { - } \quad \text { Personal que camina hasta el otro extremo de un área para recoger notas } \\
\text { - } \quad \text { Almacenes centrales para artículos de uso común, en lugar de localizarlos } \\
\text { en almacenes de artículos de mayor frecuencia de uso }\end{array}$ \\
\hline Inventario & $\begin{array}{l}\text { Inventario } \\
\text { - } \quad \text { Exceso de existencias en almacenes que no están siendo utilizadas } \\
\text { - } \quad \text { Pacientes en espera de ser dados de alta } \\
\text { - } \quad \text { Listas de espera }\end{array}$ \\
\hline Movimiento & $\begin{array}{l}\text { Movimiento } \\
\text { - } \quad \text { Movimiento innecesario de personal buscando papeles } \\
\text { - No contar con el equipo básico en cada examen/habitación }\end{array}$ \\
\hline En espera ( retardo) & $\begin{array}{l}\text { En espera } \\
\text { - } \quad \text { Pacientes, resultados, preceptos y medicamentos } \\
\text { - } \quad \text { Médicos para dar alta a los pacientes }\end{array}$ \\
\hline Sobreproducción & $\begin{array}{l}\text { Sobreproducción } \\
\text { - } \quad \text { Solicitud de pruebas diagnósticas innecesarias }\end{array}$ \\
\hline $\begin{array}{l}\text { Durante el } \\
\text { procesamiento }\end{array}$ & $\begin{array}{l}\text { Durante el proceso de atención } \\
\text { - } \quad \text { Duplicación de la información } \\
\text { - } \quad \text { Pedir información sobre los pacientes varias veces }\end{array}$ \\
\hline Defectos & $\begin{array}{l}\text { Corrección } \\
\text { - } \quad \text { Reingreso de pacientes por fallas en la atención } \\
\text { - } \quad \text { Repetición de pruebas ya que la información no era correcta }\end{array}$ \\
\hline
\end{tabular}

Fuente: (67) 
En Australia, el Hospital Flinders, con 580 camas, intervino su modelo de asignación de camas apoyado en la metodología del PE. Esta asignación se realizaba según criterios de prioridad clínica, así, los casos urgentes se asignaban a cualquier cama libre que surgiera, aunque ese piso no fuera el especializado en la patología del paciente. Esto generaba pérdidas de tiempo ya que eran equipos clínicos enteros desplazándose hasta incluso diez plantas diferentes. Ante esto el hospital cambió a un sistema de planta en pull (halar), desde donde los especialistas de cada planta ingresan a los pacientes cuando las camas quedan libres. Para responder a los casos en que la planta de una determinada patología esté totalmente ocupada, se identifica la siguiente planta más adecuada para cada tipo de paciente. Este sistema permite disminuir los desplazamientos y atenúa la falta de disponibilidad del equipo médico adecuado para la patología de cada paciente. El número de pacientes en pisos que no estaban relacionados con su patología se redujo a la mitad, el recambio de pacientes aumentó en un $20 \%$, con lo que se redujo la estancia media un día. Además, hubo mejoras en el trabajo en equipo, en la comunicación entre especialistas y en el desarrollo de habilidades del personal de enfermería necesarias para cada patología (68).

En Estados Unidos, el Centro Médico de Nebraska, con aproximadamente 620 camas, rediseñó el área de trabajo en el centro de procesamiento estéril y el laboratorio clínico, bajo los principios del PE. Con esto se logró reducir los recorridos del personal en al menos 167 kilómetros al año, así como disminuir el espacio de laboratorio por $825 \mathrm{~m}^{2}$ (de esta manera, el tiempo de procesamiento de las muestras se redujo el $20 \%$ ) y reducir la duración media de la estancia de 6,29 a 5,72 días (69).

En un estudio de tiempos y movimientos realizado en Colombia se realizó con éxito la mejora de procesos bajo la metodología del PE. En el servicio de urgencias de la Clínica Belén de Fusagasugá, entre enero del 2010 y noviembre del 2011, se modificaron los procesos con el fin de eliminar tiempos muertos y movimientos. Así, se logró una reducción en los tiempos de atención y en las quejas por la prestación de los servicios, y al mismo tiempo aumentó el giro cama, disminuyeron los días de estancia en hospitalización y el tiempo de generación de la salida del paciente de la institución, y mejoró el control del gasto al optimizarse el personal existente (69).

\section{Discusión}

Para la comprensión de la estancia hospitalaria se realizó una revisión de la literatura donde se encontró que los diferentes actores del sistema (personal de salud, administración hospitalaria, pacientes) y la relación entre entidades de la red de atención influyen en la prolongación la estancia. Según esta revisión, se considera que el factor más comúnmente encontrado dentro los hospitales que prolongan la estancia es la demora en la realización de procedimientos quirúrgicos y diagnósticos, seguido de la necesidad de atención en otro nivel de complejidad, la situación socio-familiar y la edad del paciente.

Entre las metodologías de la logística hospitalaria empleadas para abordar problemas de duración de la estancia hospitalaria se encuentran la simulación de eventos discretos, la dinámica de sistemas, la teoría de restricciones, el análisis envolvente de datos, el pensamiento esbelto, la reingeniería y los procesos de Markov.

La revisión evidencia la necesidad de desarrollar mayores niveles de eficiencia y calidad en la administración hospitalaria, de manera tal que permita la reducción de costos en 
el sistema y el acceso de la población a la atención de salud. De ahí la necesidad de que la administración hospitalaria se realice de acuerdo con un enfoque interdisciplinario. En el marco de la ingeniería con la validación de disciplinas de salud, esto ha llevado a los investigadores a incursionar en el estudio y la aplicación de diferentes metodologías como la simulación de eventos discretos, la dinámica de sistemas, el análisis envolvente de datos, la teoría de restricciones, los modelos de Markov, el pensamiento esbelto y la reingeniería.

La prolongación de la estancia de pacientes es un problema complejo y multicausal, depende de la interacción sistémica entre los servicios que intervienen en el proceso y de las características de los pacientes. Comprende la interrelación de muchos factores, con muchos enlaces de retroalimentación y demoras; por lo tanto, las causas de larga estancia pueden estar muy lejos, en tiempo y espacio, de sus efectos observados. En este caso es conveniente utilizar metodologías que permitan el estudio de sistemas complejos que cambian en función del tiempo, como la simulación.

La simulación es utilizada para hacer estimaciones del tiempo de espera de pacientes, análisis de capacidad, para evaluar las consecuencias de implementar diferentes políticas antes de ser implementadas en la práctica, para planear la capacidad, detectar cuellos de botella, agilizar los servicios que se prestan en general en las diferentes áreas del hospital y evitar congestiones (47). De tal modo, es una metodología que ofrece amplias posibilidades en la toma de decisiones para la reducción de la estancia hospitalaria, aplicable para el análisis de los diferentes tipos de factores causales encontrados en la literatura.
La simulación permite enfatizar en los puntos críticos que existen en las políticas actuales y las posibilidades de cambio que existen en ellas, sin incurrir en grandes costos, ya que permite experimentar a través del modelado en un software, el efecto esperado de un cambio previo a su implementación. Además, facilita explicar con claridad las recomendaciones a una persona que no conozca de simulación, ya que ilustra con facilidad la razón de una recomendación y el impacto esperado de su implementación, haciendo que las personas adquieran una percepción diferente de la realidad y accedan con facilidad a los cambios propuestos.

\section{Conclusiones}

Dado que la estancia hospitalaria comprende factores derivados de la condición del paciente y de la operación de los hospitales, se recomienda guiar su estudio realizando un ajuste por riesgo, para identificar cuándo la estancia prolongada está asociada a problemas de la atención y no a las características del paciente. Los GRD son el método de ajuste por riesgo más utilizado mundialmente (43, 46) y se puede acceder a este a través de software comerciales (5).

Abordar el problema de estancia prolongada en los hospitales con metodologías que no contemplen relaciones causales en la prolongación de la estancia, podría concluir en recomendaciones para implementar acciones que en el corto plazo generan beneficios, pero en el largo plazo pueden causar perjuicios en el proceso del hospital. Por lo tanto, se recomienda usar metodologías de simulación, como dinámica de sistemas, simulación de eventos discretos y simulación basada en agentes, las cuales permiten el estudio de sistemas complejos que cambian en función del tiempo. 
Como investigaciones futuras se plantea la necesidad de un estudio que evalúe el problema con un enfoque sistémico, el cual contemple variables intervenibles directamente por la administración hospitalaria como la demanda y el ingreso de pacientes, el diagnóstico, la disponibilidad de camas, los tiempos de los servicios de cirugía, ayudas diagnósticas e interconsultas y el proceso de ronda hospitalaria.

Cabe resaltar además que en los estudios revisados no se encontraron metodologías que involucraran la relación con la red de atención; se limitaban a la aplicación en el marco de los hospitales. Entonces, también se plantea la necesidad de un estudio que evalúe la interrelación de la red de atención, contemplando variables como tiempos de remisión de pacientes y tiempos en la autorización de servicios de salud.

\section{Referencias bibliográficas}

1. García A, Ramos I, García J, Gálvez A. El balance entre la oferta y la demanda en salud. El caso de los servicios de rehabilitación integral en La Habana, Cuba. Escuela Nacional de Salud Pública; 20092010 [Internet]. Disponible en: http://bvs.sld.cu/ revistas/infd/n1211/infd061211.htm

2. Afanador G. Atención segura y de calidad al paciente a través del manejo del proceso hospitalario. Revista Inalde [Internet]. Disponible en: http:// www.inalde.edu.co/sala-de-prensa/revista-inalde/ detalle-blog/ic/atencion-segura-y-de-calidad-alpaciente-a-traves-del-manejo-del-proceso-hospitalario/icac/show/Content/

3. Correa A. Baja capacidad hospitalaria. Periódico El Colombiano, septiembre del 2010 [Internet]. Disponible en: http://www.elcolombiano.com/ BancoConocimiento/B/baja_capacidad_hospitalaria/baja_capacidad hospitalaria.asp

4. Perales P, Amores P, Escrivá R, Pastor A, Alvarruiz J, De la Calzada J. Adecuación de los ingresos hospitalarios no quirúrgicos desde un servicio de urgencias. Revista Emergencias. 2004; 16: 111-5.

5. Jiménez R. Indicadores de calidad y eficiencia de los servicios hospitalarios. Una mirada actual. Revista Cubana Salud Pública. 2004; 30 (1).

6. González Angulo I. Relación entre el prestador de servicio de salud y la estancia prolongada en el hospital. Revista Conamed. 2009; 14 (4).
7. Kim C, Hart A, Paretti R, Kuhn L, Dowling A, Benkeser J et ál. Excess Hospitalization Days in an Academic Medical Center: Perceptions of Hospitalists and Discharge Planners. The American Journal of Managed Care. 2011; 17 (2).

8. Carencias del sistema hospitalario colombiano. Periódico El Pulso, diciembre del 2007 [Internet]. Disponible en: http://www.periodicoelpulso.com/ html/0712dic/general/general-12.htm

9. Morgan M, Beech R. Variations in Lengths of Stay and Rates of Day Case Surgery: Implications for the Efficiency of Surgical Management. Journal of Epidemiology and Community Health. 1979; 44 (2): 90-105.

10. Varela A, Carrasquilla G. La estancia hospitalaria, un trazador de la atención. Foro permanente La calidad de la atención de la salud, Academia Nacional de Medicina de Colombia y Universidad del Valle; 1999.

11. Palacios AU, Bareño S. Factores asociados a eventos adversos en pacientes hospitalizados en una entidad de salud en Colombia. Rev CEs Med 2012; 26 (1): 19-28.

12. Gamarra P. Consecuencias de la hospitalización en el anciano. Boletín de la Sociedad Peruana de Medicina Interna. 2001; 14 (2).

13. García M, Lardelli P, Jiménez J, Bueno A, Luna J, Gálvez R. Proportion of Hospital Deaths Potentially Attributable to Nosocomial Infection. Infection Control and Hospital Epidemiology. 2001; 22 (11): 708-14.

14. Tell W, Nataraja S. Next-Generation Capacity Management. Collaborating for Clinically Appropriate and Efficient Inpatient Throughput. Advisory Board International; 2010.

15. Negro J, Jiménez J, Félix R, Pascual A, Miralles J, López $\mathrm{J}$ et ál. Adecuación de ingresos y estancias hospitalarias en pacientes con asma bronquial. Mejora de la gestión de las camas en la sección de alergología, tras la difusión de un estudio previo. Alergol Inmunol Clin. 2003; 18: 61-70.

16. Ríos J, Fazzari P, Lugano I. Utilización hospitalaria inadecuada en servicios clínicos. Una experiencia con el AEP en un Hospital Universitario de la República Argentina; 2002.

17. Ferreira A, Rígoli F, Bazzani R. Procesos asociados con el tiempo de estadía en el Hospital de Clínicas: estudio en cuatro enfermedades trazadoras. Revista Médica del Uruguay. 1997; 13: 23-36.

18. Elorza M, Vanina N, Cruciani F, Moscoso N, Gullacei M. Clasificación de las causas que determinan estadía inadecuada útil para la gestión hospitalaria. Consejo Nacional de Investigaciones Científicas y Técnicas (Conicet) de Argentina, Universidad Nacional del Sur, Argentina; 2009.

19. Pérez A, Santos S, Luquero F, Tamames S, Cantón B, Castrodeza J. Evaluación de la adecuación de las estancias en un hospital de tercer nivel. An Sist Sanit Navar. 2007; 30 (1).

20. San Román J, Luquero F, Fuente L, Pérez A, Tamames S, Fernández F, Castrodeza J. Evaluación de las estancias inadecuadas en un servicio 
de cardiología. Revista Española de Cardiología, 2009; 62 (2): 211-5.

21. Irfan S. Hospitalist: Long Hospital Stays Are often Due to Poor Planning. Kevin MD [Serie en internet]; 2010. Disponible en: http://www.kevinmd. com/blog/2010/09/hospitalist-long-hospital-staysdue-poor-planning.html.

22. Caminiti C, Meschi T, Braglia L, Diodati F, Iezzi E, Marcomini B et ál. Reducing Unnecessary Hospital Days to Improve Quality of Care through Physician Accountability: A Cluster Randomised Trial. BioMed Central Health Services Research. 2013; 13.

23. Zonana A, Baldenebro R, Felix G, Gutiérrez P. Tiempo de estancia en medicina interna: Función del médico hospitalista. Rev Med Inst Mex Seguro Soc. 2011; 49 (5): 527-31.

24. Mould J, García C, Contreras I, Juárez T, Espinel C, Morales G et ál. Direct Costs Associated with the Appropriateness of Hospital Stay in Elderly Population. BMC Health Services Research. 2009; 9 [Internet]. Disponible en: http://www.biomedcentral.com/1472-6963/9/151.

25. Alfaro L. Redefinición de estándares de las estancias promedio hospitalarias según complejidad de la morbilidad en los servicios de medicina y cirugía del hospital San Rafael de Alajuela, análisis de causas de incumplimiento y planteamiento de mejoras [tesis de especialización en Administración de Servicios de Salud]. Instituto Centroamericano de Administración Pública (ICAP), Costa Rica; 2008.

26. Perales P, Amores P, Escrivá R, Pastor A, Alvarruiz J, De la Calzada J. Adecuación de los ingresos hospitalarios no quirúrgicos desde un servicio de urgencias. Revista Emergencias. 2004; 16: 111-5.

27. DeCoster C, Kozyrskyj A. Long-Stay Patients in Winnipeg Acute Care Hospitals. Manitoba Centre for Health Policy and Evaluation. Faculty of Medicine, University of Manitoba; 2000.

28. Almeida A, Serraqueiro Z, Rogeiro A. Protocolo de revisão de utilização de recursos num hospital público português. Acta Med Port. 2006; 19: 381-5.

29. Vilaróa J, Yáñez A, Casadevalla J, Sanclementea C, Parceta A, Barconsa M et ál. Adecuación de la estancia hospitalaria en un servicio de medicina interna de un hospital comarcal. Revista Calidad Asistencial. 2003; 18 (8): 625-7.

30. Isherwood J, Howard D, Saunders R, Jabri Y, Phillips D, McWhinnie D. What Factors Are Associated with Prolonged Hospital Stay Following Planned Day-Case. Laparoscopic Cholecystectomy? Ambulatory Surgery. 2013; 19 (1).

31. Zambrana J, Delgado M, Cruz G, Martín M, Díez F, Ruiz M. Factores predictivos de estancias no adecuadas en un servicio de medicina interna. Medicina Clínica. 2001; 117 (3).

32. Aledo S, Carrillo A, Campillo Á, Fernández M, Leal J, Flores B et ál. Impacto de las medidas de intervención para disminuir estancias hospitalarias innecesarias. Revista Gestión y Evaluación de Costes Sanitarios. 2009; 10 (2).

33. Carey M, Heena S, Scott R. Un estudio prospectivo de motivos de hospitalizaciones prolongadas en un servicio de medicina general docente. J Gen Intern Med. 2005 Feb; 20 (2): 108-15.

34. Disminución de estancias inactivas en la Clínica Iss de Envigado, Antioquia. Gerencia Nacional de Calidad; 2001.

35. Velasco L, García S, Oterino D, Suárez F, Diego $\mathrm{S}$, Reyes A. Impacto de los ingresos urgentes innecesarios sobre las estancias hospitalarias en un hospital de Asturias. Revista España Salud Pública. 2005; 79: 541-9.

36. Lamberth J, Gooskens M, Verheggen F, Pop P, Prins M. Predictors of Inappropiate Hospital Stay: A Clinical Case Study. International Journal for Quality in Health Care. 2003; 15 (1): 57-65.

37. Merom D, Shohat T, Harari G, Meir O, Green M. Factors Associated with Inappropriate Hospitalization Days in Internal Medicine Wards in Israel: A Cross-National Survey. International Journal for Quality in Health Care. 1998; 10 (2): 155-62.

38. Silber JH, Rosenbaum P, Rosen A, Romano P, Itani K, Cen L et ál. Prolonged Hospital Stay and the Resident Duty Hour Rules of 2003. Med Care. 2009 Dic; 47 (12): 1191-00.

39. Hendy P, Patel JH, Kordbacheh T, Laskar N, Harbord M. In-Depth Analysis of Delays to Patient Discharge: A Metropolitan Teaching Hospital Experience. Clinical Medicine. 2012; 12 (4): 320-3.

40. Berki S, Ashcraft MLF, Newbrander W. Lengthof-Stay Variations within ICDA-8 Diagnosis-Related Groups. Medical Care. 1984 Feb; 22 (2): 126-142.

41. Parker M, Todd C, Palmer C, Camilleri C, Freeman C, Laxton C, Payne B, Rushton N. Inter-Hospital Variations in Length of Hospital Stay Following Hip Fracture. Age and Ageing. 1998; 27: 333-7.

42. Bastero C, Martínez L, Olivé M. La optimización de los circuitos hospitalarios en la gestión de camas [trabajo en Máster en Administración y Gestión en Cuidados de Enfermería]. Escuela Universitaria de Enfermería Santa Madrona; 2009.

43. Tamargo T, Jiménez R, Gutiérrez A, Mora I. Dos procedimientos de ajuste por riesgo para la estadía hospitalaria como indicador de desempeño. Revista Cubana de Salud Pública. 2012; 38 (1): 29-44.

44. Lim S, Doshi V, Castasus B, Lim JKH, Mamun K. Factors Causing Delay in Discharge of Elderly Patients in an Acute Care Hospital. Changi General Hospital. Annals Academy of Medicine. 2006; 35 (1).

45. Toshikazu A, Yasuharu T, Stein G, Ishimatsu S, Birrer R. Risk Factors Associated with Prolonged Hospital Stay in Admitted Patients with Psychotropic Drug Overdose. The Open Critical Care Medicine Journal. 2008; 1: 12-16.

46. González V, Maciá S. Grupos de pacientes relacionados por el diagnóstico (GRD) en los hospitales generales españoles: variabilidad en la estancia media y el coste medio por proceso. Revista Enfermería Global. 2011 Oct; 24: 126-43.

47. Jiménez A, Guerrero J, Amaya C, Velasco N. Optimización de los recursos en los hospitales: revisión de la literatura sobre logística hospitalaria. Los cuadernos de PYLO - Logística Hospitalaria. Bogotá: Universidad de los Andes; 2007. 
48. Velásquez P, Rodríguez A, Jaén J. Metodologías cuantitativas para la optimización del servicio de urgencias: una revisión de la literatura. Revista Gerencia y Políticas de Salud. 2011; 10 (21): 196-18.

49. Kolker A. Queuing Analytic Theory and Discrete Events Simulation for Healthcare: Right Application for the Right Problem [Internet]. Disponible en: http://www.researchgate.net/publication/255601401_Queuing_Analytic_Theory_ and_Discrete_Events_Simulation_for_Healthcare_Right_Application_for_the_Right_Problem.

50. El-Darzi E, Vasilakis $\bar{C}$, $\bar{C}$ haussalet $\bar{T}$, Millard P. A Simulation Modelling Approach to Evaluating Length of Stay, Occupancy, Emptiness and Bed Blocking in a Hospital Geriatric Department. Health Care Management Science. 1998; 1: 143-9.

51. Williams P, Tai G, Lei Y. Simulation Based Analysis of Patient Arrival to Health Care Systems and Evaluation of an Operations Improvement Scheme. Department of Manufacturing and Operations Engineering, University of Limerick, Ireland. Ann Oper Res. 2010; 178: 263-79.

52. Wolstenholme E. Emerging Opportunities for System Dynamics in UK Health and Social care - The Market- Pull for Systemic Thinking. En: International Conference of the System Dynamics Society $\left(26^{\circ}, 2008\right.$, Grecia). Actas de la $26^{\circ}$ Conferencia Internacional de la Sociedad de Dinámica de Sistemas; 2008.

53. Cooke D. Introducing System Dynamics Modeling to Health Care in Alberta. Submitted to the $25^{\text {th }}$ International Conference of the System Dynamics Society, Boston, Estados Unidos, 2007.

54. Ahmad N, Abdul N, Abdulbasah A. A Framework for Emergency Department Capacity Planning Using System Dynamics Approach and the Theory of Constraints Philosophies. Proceedings $24^{\text {th }} \mathrm{Eu}-$ ropean Conference on Modelling and Simulation, Kuala Lumpur, Malasia, junio 1-4 del 2010.

55. Breen A, Burton-Houle T, Aron D. Applying the Theory of Constraints in Health Care: Part 1 - The Philosophy. Quality Management in Health Care. 2002; 3 (3).

56. Wright J, King R. We All Fall Down: Goldratt's Theory of Constraints for Healthcare Systems. Great Barrington, Mass.: North River Press, The New England Journal of Medicine; 2006.

57. Knight A. Alcanzando un desempeño superior a través de todo el sistema de salud y seguridad social. Traducción y comentarios: Conde R, Hernández L. 2004 [Internet]. Disponible en: http://www.e-toc.com/-/index.php?option =com content \&view $=$ article $\&$ id $=56 \% 3$ Atocsalud \& catid $=25 \&$ Itemid $=600005$

58. Aguirre N. Applying Theory of Constraints to Improve Performance in a Hospital without Increase the Costs [Master in business direction and management]. Bogotá: Universidad del Rosario; 2008.

59. Prior D, Solá M. Listas de espera en los hospitales públicos españoles y acciones estratégicas para su control. Revista oIKOs. 2007; 11 (24).

60. Carrada T. La reingeniería en sistemas de salud. Rev Med IMSs. 2002; 40 (2): 127-35.
61. Villa S, Barbieri M, Lega F. Restructuring Patient Flow Logistics around Patient Care Needs: Implications and Practicalities from Three Critical Cases. Health Care Manag Sci. 2009; 12: 155-65.

62. Ardila S, Jiménez AM, Velasco N, Amaya C. Revisión de procesos para la asignación de camas a pacientes provenientes de urgencias en un hospital privado de Bogotá. Los cuadernos de PYLO - Logística Hospitalaria. Bogotá: Universidad de los Andes; 2008.

63. Albornoz V, Hinrichsen M, Miranda P, Peña P. Uso de cadenas de Markov para la predicción de la dinámica del comportamiento de pacientes en una unidad de cuidado intensivo cardiológica. Ingeniare - Revista Chilena de Ingeniería. 2006; 14 (2), 153-8.

64. Nadal L, De Carvalho S, De Cassia R. Markov Decision Process Applied to the Control of Hospital Elective Admissions. Artificial Intelligence in Medicine. 2009; 47: 159-71.

65. Peguero R, Riquenes G, Caballero G. Aplicación de las cadenas de Markov a los procesos de servicios hospitalarios para la toma de decisiones en la administración de la salud. Observatorio de la Economía Latinoamericana. 2011; 143 [Internet]. Disponible en: http://www.eumed.net/cursecon/ecolat/cu/2011/

66. Hincapié P, Ospina J, Grisales H, Arroyave M, Valencia M, González E. Análisis markoviano de un proceso de estancia hospitalaria en un hospital de tercer nivel de complejidad. Revista Facultad Nacional de Salud Pública. 2004; 22 (1): 61-72.

67. Radnor Z, Holweg M, Waring J. Lean in Healthcare: The Unfilled Promise? Social Science \& Medicine. 2012; 74: 364-71.

68. Jones D, Mitchell A. Lean Thinking for the NHS. A Report Commissioned by the NHS Confederation [Internet]. Disponible en: http://www.leanuk.org/ downloads/health/lean_thinking_for_the_nhs_leaflet.pdf

69. Palomino A, Guarín H. Aplicación de los modelos de mejoramiento de procesos y de tiempos y movimientos en las áreas de urgencias y hospitalización de la Clínica Belén de Fusagasugá para garantizar la prestación del servicio en salud con calidad [tesis de Maestría en Administración en Salud]. Bogotá: Universidad del Rosario; 2012.

70. Banks J, Carson J, Nelson B, Nicol D. DiscreteEvent System Simulation, cuarta edición. Pearson; 2005.

71. Günal M, Pidd M. Simulación de eventos discretos para modelar el desempeño en la atención de la salud: una revisión de la literatura. Lancaster University Management School, Reino Unido; 2009.

72. Forrester JW. Industrial Dynamics. Waltham, MA: Pegasus Communications; 1961.

73. VanBerkel P, Blake T. A Comprehensive Simulation for Wait Time Reduction and Capacity Planning Applied in General Surgery. Health Care Manage Sci. 2007; 10: 373-85.

74. Sedano H. ¿Qué es Toc? e-Toc [Internet]. Disponible en: http://e-toc.com/-/?option $=$ com content\&task $=$ view\&id $=30$

75. López I, Urrea J, Navarro D. Aplicación de la Teoría de Restricciones (тос) a la gestión de fac- 
turación de las Empresas Sociales del Estado, ESE. Una contribución al Sistema de Seguridad Social en Colombia. Revista Innovar. 2006; 16 (27).

76. León Y. Aplicación de la teoría de restricciones en la gestión de la seguridad del paciente [tesis de Maestría en Administración en Salud]. Bogotá: Facultad de Administración de la Universidad del Rosario; 2012.

77. Polanía B. Una aplicación de data envelopment analysis: análisis comparativo de eficiencia entre hospitales en Colombia. [tesis de Maestría en Ingeniería Industrial]. Bogotá: Universidad de los Andes; 1999.

78. Rubio B, Rubio S, Repullo J. En busca de nuevas herramientas de análisis de la eficiencia en el sector público sanitario. Revista Administración Sanitaria (Escuela Nacional de Sanidad, Instituto de Salud Carlos III, Madrid). 2007; 5 (4): 659-72.

79. Powell A, Rushmer R, Davies HTO. A Systematic Narrative Review of Quality Improvement Models in Health Care (in support of NHs Quality Improvement Scotland). Social Dimensions of Health Institute at The Universities of Dundee and St Andrews; 2008.
80. Osorio J, Paredes E. Reingeniería de procesos en los hospitales públicos: ¿reinventando la rueda? Revista Española de Salud Pública. 2001; 75: 193-06.

81. Albizu E, Olazaran M, Simon K. La reingeniería como programa de cambio organizativo. Revista de Economía y Empresa. 2004; 21 (51).

82. Ortiga B. Reingeniería de los procesos de ingreso y alta para una gestión eficiente. Hospital Universitario de Vellbitge; 2008.

83. Ocaña R. Modelos de Markov aplicados a la investigación en Ciencias de la Salud. Interciencia. 2009; 34 (3).

84. Planificación de métodos estadísticos en ciencias de la vida. Programa de la Universidad Nacional de Entre Ríos, Argentina.

85. Carrillo M, Pons R, Barrios P, Puello M. Lean Thinking, metodología de gestión de mejoramiento en instituciones de educación superior. Eighth LACCEI Latin American and Caribbean Conference for Engineering and Technology (LACCEI'2010) "Innovation and Development for the Americas", Junio 1-4, 2010, Arequipa, Perú. 